\title{
An algorithm which generates linear extensions for a non-simply-laced d-complete poset with uniform probability
}

\author{
Kento Nakadali \\ Graduate School of Education Master's Program, Okayama University, Japan
}

\begin{abstract}
The purpose of this paper is to present an algorithm which generates linear extensions for a non-simplylaced d-complete poset with uniform probability.

Résumé. Le but de ce papier est présenter un algorithme qui produit des extensions linéaires pour une non-simplylaced d-complete poset avec probabilité constante.
\end{abstract}

Keywords: d-complete posets, algorithm, linear extension, uniform generation

\section{Introduction}

In [7](Theorem 4.2), J. Stembridge classified irreducible minuscule elements of Kac-Moody Weyl group over a root system $\Phi$ into three classes below:

- $\Phi$ is simply-laced,

- $\Phi$ has the form

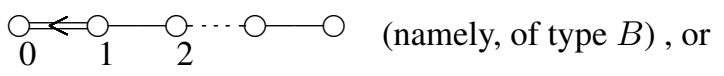

- $\Phi$ has the form

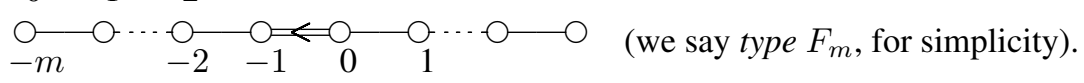

In [5][6], the author and S. Okamura constracted an algorithm which generates reduced decompositions for a given minuscule element of simply-laced Weyl group with uniform probability. The algorithm in [6] is described in terms of graphs. Simply-laced minuscule elements are described as certain simple acyclic di-graphs. The transitive-closure of the graph is called a d-complete poset. Then, the reduced decompositions are identified with linear extensions of the graph. This algorithm gives a proof of the hook formula [1] for the number of reduced decompositions of a minuscule element in simply-laced case.

In this paper, we present an algorithm (algorithm A) in terms of graphs (See Section 2 for details). This algorithm is a generalization of an algorithm in [5][6]. We define a certain acyclic multi-di-graph corresponding to a minuscule element of type $B$ (resp. type $F_{m}$ ) in Section 3 (resp. Section 4). Our

\footnotetext{
${ }^{\dagger}$ Email: nakada@okayama-u.ac.jp 
main result (Theorem 5.1) is that the algorithm A generates linear extensions for a minuscule element of type $B$ and $F_{m}$ with uniform probability. More precisely, the probability the algorithm A generates linear extension $L$ of a graph $S$ is given by:

$$
\frac{\prod_{v \in S}\left(1+\# \mathrm{H}_{S}(v)^{+}\right)}{\# S !}
$$

where $\mathrm{H}_{S}(v)^{+}$is a certain subset of $S$ (See Section 2 for detail). This 1.11 is independent from the choice of $L$. Hence, we get the hook formula for the number of linear extensions of a given shape $S$ of type $B$ and $F_{m}$. Namely, the number of linear extensions of a shape $S$ is given by:

$$
\frac{\# S !}{\prod_{v \in S}\left(1+\# \mathrm{H}_{S}(v)^{+}\right)} .
$$

In section 6, we give a Lie theoretical description of shape of type $B$ and $F_{m}$.

\section{An algorithm for a graph $\Gamma$}

Let $\Gamma=(\Gamma ; A, \mathrm{o}, \mathrm{i})$ be a finite acyclic multi-di-graph, where $A$ denotes the set of arrows of $\Gamma, \mathrm{i}(a)$ the sink of $a \in A$, and o $(a)$ the source of $a \in A$.

Definition 2.1 Put $d:=\# \Gamma$. A bijection $L:\{1, \cdots, d\} \longrightarrow \Gamma$ is said to be a linear extension of $\Gamma$ if:

$$
L(k)=\mathrm{o}(a) \text { and } \mathrm{i}(a)=L(l) \text { implies } k>l, \quad k, l \in\{1, \cdots, d\}, \quad a \in A .
$$

The set of linear extensions of $\Gamma$ is denoted by $\mathcal{L}(\Gamma)$.

For a given $v \in \Gamma$, we define a set $\mathrm{H}_{\Gamma}(v)^{+}$by:

$$
\mathrm{H}_{\Gamma}(v)^{+}:=\{a \in A(\Gamma) \mid v=\mathrm{o}(a)\} .
$$

For a given $\Gamma$, we call the following algorithm the algorithm $\mathrm{A}$ for $\Gamma$ :

GNW1. Set $i:=0$ and set $\Gamma_{0}:=\Gamma$.

GNW2. (Now $\Gamma_{i}$ has $d-i$ nodes.) Set $j:=1$ and pick a node $v_{1} \in \Gamma_{i}$ with the probability $1 /(d-i)$.

GNW3. If $\# \mathrm{H}_{\Gamma_{i}}\left(v_{j}\right)^{+} \neq 0$, pick an arrow $a_{j+1} \in \mathrm{H}_{\Gamma_{i}}\left(v_{j}\right)^{+}$with the probability $1 / \# \mathrm{H}_{\Gamma_{i}}\left(v_{j}\right)^{+}$. If not, go to GNW5.

GNW4. Set $v_{j+1}:=\mathrm{i}\left(a_{j}\right)$. Set $j:=j+1$ and return to GNW3.

GNW5. (Now $\# \mathrm{H}_{\Gamma_{i}}\left(v_{j}\right)^{+}=0$.) Set $L(i+1):=v_{j}$ and set $\Gamma_{i+1}:=\Gamma_{i} \backslash v_{j}$ (the graph deleted $v_{j}$ from $\Gamma_{i}$ ).

GNW6. Set $i:=i+1$. If $i<d$, return to GNW2; if $i=d$, terminate.

We note that the algorithm A stops in finite time since $\Gamma$ is acyclic. By the definition of the algorithm A for $\Gamma$, the map $L: i \mapsto L(i)$ generated above is a linear extension of $\Gamma$. We denote by $\operatorname{Prob}_{\Gamma}(L)$ the probability we get $L \in \mathcal{L}(\Gamma)$ by the algorithm A. 
An algorithm which generates linear extensions for a non-simply-laced d-complete poset with uniform probability 657

\section{Shapes of type $B$}

We denote by $\mathbb{N}$ the set of non-negative integers. We define a set $\mathbb{B}$ by:

$$
\mathbb{B}:=\{(i, j) \in \mathbb{N} \times \mathbb{N} \mid i \leq j\} .
$$

The set $\mathbb{B}$ is depicted in FIGURE 3.1. We equip the $\mathbb{B}$ with the partial order:

$$
(i, j) \leq\left(i^{\prime}, j^{\prime}\right) \Longleftrightarrow i \geq i^{\prime} \text { and } j \geq j^{\prime} .
$$

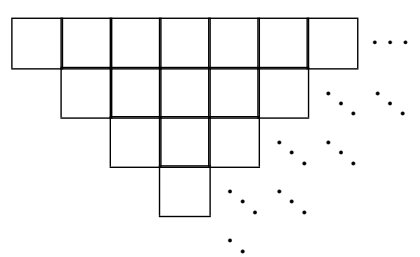

Fig. 3.1: The set $\mathbb{B}$

Definition 3.1 Let $S$ be a finite order filter of $\mathbb{B}$. We induce to $S$ a graph structure by:

$$
(i, j) \rightarrow\left(i^{\prime}, j^{\prime}\right) \text { if and only if }\left\{\begin{array}{c}
\text { " } i=j \text { and } i^{\prime}=i, j^{\prime}>j ”, \\
" i<j \text { and } i^{\prime}=i, j^{\prime}>j ”, \\
\text { " } i<j \text { and } i^{\prime}>i, j^{\prime}=j ”, \\
\text { or " } i<j \text { and } i^{\prime}=j, j^{\prime}>i ”,
\end{array}\right.
$$

and there exists no other adjacency relation. Here, $v \rightarrow v^{\prime}$ means there exists exactly one arrow from $v$ to $v^{\prime}$, and $v \rightrightarrows v^{\prime}$ there exists exactly two arrows from $v$ to $v^{\prime}$. A graph $S$ is called a shape of type B. See FIGURE 3.2 for examples of $\mathrm{H}_{S}(v)^{+}$.
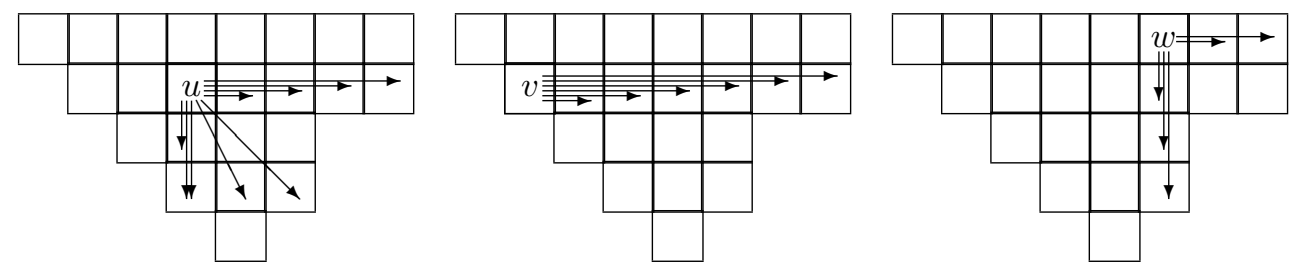

Fig. 3.2: $\mathrm{H}_{S}(u)^{+}, \mathrm{H}_{S}(v)^{+}$, and $\mathrm{H}_{S}(w)^{+}$.

Remark 3.2 A shape of type B as poset is order-isomorphic to a shifted shape. Shifted shapes are also realized as d-complete posets over a root system of type D. The graph-structure of shapes of type $D$ is described in [6] and compatible with notion of hooks (or called bars) of shifted shapes. The algorithm A depends not only on poset-structure but on graph-structure. Hence, we do not consider shapes of type B as shifted shapes. 


\section{Shapes of type $F_{m} \quad(m \geq 2)$.}

We denote by $\mathbb{Z}$ the set of integers. Let $m$ be an integer greater than or equal to 2 . We define a set $\mathbb{F}_{m}$ by:

$$
\mathbb{F}_{m}:=\left\{\begin{array}{l|l}
(i, j) \in \mathbb{N} \times \mathbb{Z} & \begin{array}{l}
i=0 \text { and } j \geq-m, \\
i=1 \text { and } j \geq 0, \text { or } \\
2 \leq i \leq m \text { and } j=0
\end{array}
\end{array}\right\}
$$

For example, the set $\mathbb{F}_{3}$ is depicted in FIGURE 4.1. We equip the $\mathbb{F}_{m}$ with the partial order:

$$
(i, j) \leq\left(i^{\prime}, j^{\prime}\right) \Longleftrightarrow i \geq i^{\prime} \text { and } j \geq j^{\prime} .
$$

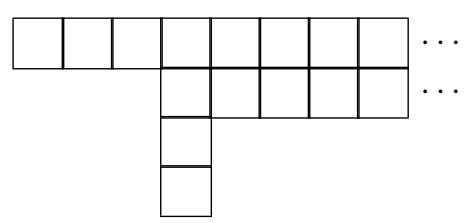

Fig. 4.1: The set $\mathbb{F}_{3}$

Definition 4.1 Let $S$ be a finite order filter of $\mathbb{F}_{m}$. We induce to $S$ a graph structure by:

$$
\begin{aligned}
& \text { " } i=0, j \leq-1 \text { and } i^{\prime} \neq-j, j^{\prime}>j \text {, } \\
& \text { " } i=0, j=0 \text {, and } j^{\prime}>0 \text { ", } \\
& " i=1, j=0 \text {, and } i^{\prime}=1, j^{\prime}>0 ", \\
& (i, j) \rightarrow\left(i^{\prime}, j^{\prime}\right) \quad \text { if and only if } \quad \text { " } i=1, j=0, \text { and } i^{\prime}>1, j^{\prime}=0 \text { ”, } \\
& \text { " } i \geq 2, j=0 \text {, and } i^{\prime}>i, j^{\prime}=0 \text { ", } \\
& \text { " } j \geq 1 \text { and } i^{\prime}=i, j^{\prime}>j ", \\
& \text { or " } j \geq 1 \text { and } i^{\prime}>i, j^{\prime}=j \text { ", } \\
& (i, j) \rightrightarrows\left(i^{\prime}, j^{\prime}\right) \quad \text { if and only if } \quad \text { “ } i=0, j=0 \text {, and } 0<i^{\prime}, j^{\prime}=0 \text { ”, }
\end{aligned}
$$

and there exists no other adjacency relation. A graph $S$ is called a shape of type $F_{m}$. See FIGURE 4.2 for examples of $\mathrm{H}_{S}(v)^{+}$.
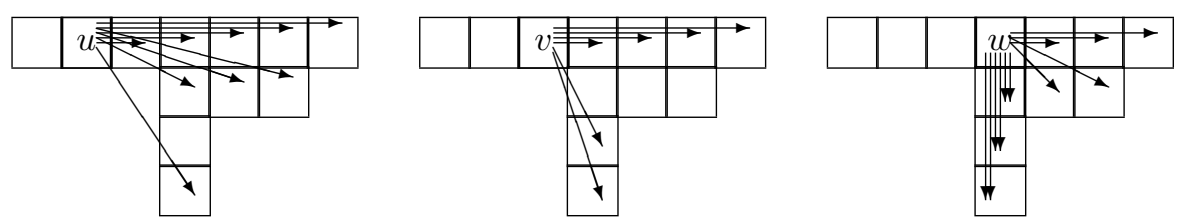

Fig. 4.2: $\mathrm{H}_{S}(u)^{+}, \mathrm{H}_{S}(v)^{+}$, and $\mathrm{H}_{S}(w)^{+}$. 
An algorithm which generates linear extensions for a non-simply-laced d-complete poset with uniform probability 659

\section{Main result}

Now, we can state the main theorem:

Theorem 5.1 Let $S$ be a shape of type $B$ or type $F_{m}$ for some $m \geq 2$. Let $L \in \mathcal{L}(S)$. Then the algorithm A for $S$ generates $L$ with the probability

$$
\operatorname{Prob}_{S}(L)=\frac{\prod_{v \in S}\left(1+\# \mathrm{H}_{S}(v)^{+}\right)}{\# S !} .
$$

Since the right hand side of (5.1) is independent from the choice of $L \in \mathcal{L}(S)$, we have:

Corollary 5.2 Let $S$ be a shape of type $B$ or type $F_{m}$ for some $m \geq 2$. Then we have:

$$
\# \mathcal{L}(S)=\frac{\# S !}{\prod_{v \in S}\left(1+\# \mathrm{H}_{S}(v)^{+}\right)} .
$$

\section{Lie theoretical description of main result and Remarks}

In this section, we fix a (not necessary simply-laced) Kac-Moody Lie algebra $\mathfrak{g}$ with a simple root system $\Pi=\left\{\alpha_{i} \mid \in I\right\}$. For all undefined terminology in this section, we refer the reader to [2] [3].

Definition 6.1 An integral weight $\lambda$ is said to be pre-dominant if:

$$
\left\langle\lambda, \beta^{\vee}\right\rangle \geq-1 \quad \text { for each } \beta^{\vee} \in \Phi_{+}^{\vee},
$$

where $\Phi_{+}^{\vee}$ denotes the set of positive real coroots. The set of pre-dominant integral weights is denoted by $P_{\geq-1}$. For $\lambda \in P_{\geq-1}$, we define the set $\mathrm{D}(\lambda)^{\vee}$ by:

$$
\mathrm{D}(\lambda)^{\vee}:=\left\{\beta^{\vee} \in \Phi_{+}^{\vee} \mid\left\langle\lambda, \beta^{\vee}\right\rangle=-1\right\} .
$$

The set $\mathrm{D}(\lambda)^{\vee}$ is called the shape of $\lambda$. If $\# \mathrm{D}(\lambda)^{\vee}<\infty$, then $\lambda$ is called finite.

Proposition 6.2 (see [4]) Let $\lambda \in P_{\geq-1}$ be finite and $\beta^{\vee}, \gamma^{\vee} \in \mathrm{D}(\lambda)^{\vee}$ satisfy $\beta^{\vee}>\gamma^{\vee}$ in the ordinary order of coroots. Then we have:

$$
\left\langle\beta, \gamma^{\vee}\right\rangle=0,1 \text {, or } 2 \text {. }
$$

By proposition 6.2, we introduce graph-structure into $\mathrm{D}(\lambda)^{\vee}$ by:

$$
\begin{aligned}
& \beta^{\vee} \rightarrow \gamma^{\vee} \Leftrightarrow \beta^{\vee}>\gamma^{\vee} \text { and }\left\langle\beta, \gamma^{\vee}\right\rangle=1 . \\
& \beta^{\vee} \rightrightarrows \gamma^{\vee} \Leftrightarrow \beta^{\vee}>\gamma^{\vee} \text { and }\left\langle\beta, \gamma^{\vee}\right\rangle=2 .
\end{aligned}
$$

If $\beta^{\vee} \ngtr \gamma^{\vee}$, or $\beta^{\vee}>\gamma^{\vee}$ and $\left\langle\beta, \gamma^{\vee}\right\rangle=0$, then no arrows from $\beta^{\vee}$ to $\gamma^{\vee}$ exist.

Thus, we get a finite acyclic multi-di-graph $\mathrm{D}(\lambda)^{\vee}$ for a finite $\lambda \in P_{\geq-1}$.

Remark 6.3 The finite pre-dominant integral weights $\lambda$ are identified with the minuscule elements $w$ [4]. And, we have $\mathrm{D}(\lambda)^{\vee}=\left\{\beta^{\vee} \in \Phi_{+}^{\vee} \mid w^{-1}\left(\beta^{\vee}\right)<0\right\}$. Furthermore, the linear extensions of $\mathrm{D}(\lambda)^{\vee}$ are identified with the reduced decompositions of $w[4]$ by the following one-to-one correspondence:

$\operatorname{Red}(w) \ni\left(s_{i_{1}}, s_{i_{2}}, \cdots, s_{i_{d}}\right) \longleftrightarrow L \in \mathcal{L}\left(\mathrm{D}(\lambda)^{\vee}\right), \quad L(k)=s_{i_{1}} \cdots s_{i_{k-1}}\left(\alpha_{i_{k}}\right)^{\vee} \in \mathrm{D}(\lambda)^{\vee}(k=1, \cdots d)$, where $\operatorname{Red}(w)$ denotes the set of reduced decompositions of $w, d=\ell(w)$ the length of $w$. 


\subsection{Case of type $B$}

Suppose that the underlying Dynkin diagram is of type $B$ :

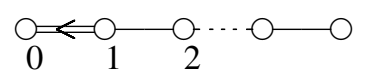

Let $W=\left\langle s_{0}, s_{1}, s_{2}, \cdots\right\rangle$ be the Weyl group. Let $\Lambda_{0}$ be the 0 -th fundamental weight. Then each $\lambda \in W \Lambda_{0}$ is a finite pre-dominant integral weight. And, $\mathrm{D}(\lambda)^{\vee}$ is graph-isomorphic with some shape of type $B$ defined in section 3 .

Remark 6.4 Let $W_{0}:=\left\langle s_{1}, s_{2}, \cdots\right\rangle$ be a maximal parabolic subgroup of $W$, which is the Weyl group of type A. Then a minimal coset representative $w$ in $W / W_{0}$ is called a Lagrangian Grassmannian element.

Let $\lambda \in W \Lambda_{0}$. Then the corresponding minuscule element $w$ in remark 6.3 is a Lagrangian Grassmannian element. Our result gives the number of reduced decompositions of Lagrangian Grassmannian element $w$.

\subsection{Case of type $F_{m}(m \geq 2)$}

Let $m \in \mathbb{Z}$ be greater than or equal to 2. Suppose that the underlying Dynkin diagram is of type $F_{m}$ :

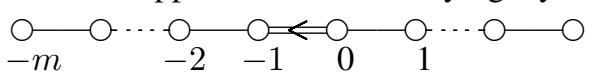

Let $W=\left\langle s_{-m}, \cdots, s_{-2}, s_{-1}, s_{0}, s_{1}, \cdots\right\rangle$ be the Weyl group. Let $\Lambda_{-m}$ be the $(-m)$-th fundamental weight. Then each $\lambda \in P_{\geq-1} \cap W \Lambda_{-m}$ is a finite pre-dominant integral weight. And, $\mathrm{D}(\lambda)^{\vee}$ is graphisomorphic with some shape of type $F_{m}$ defined in section 4 .

\section{References}

[1] J. B. Carrell, Vector fields, flag varieties and Schubert calculus, Proc. Hyderabad Conference on Algebraic Groups (ed. S.Ramanan), Manoj Prakashan, Madras, 1991.

[2] V. G. Kac, "Infinite Dimentional Lie Algebras," Cambridge Univ. Press, Cambridge, UK, 1990.

[3] R. V. Moody and A. Pianzola, "Lie Algebras With Triangular Decompositions," Canadian Mathematical Society Series of Monograph and Advanced Text, 1995.

[4] K. Nakada, Colored hook formula for a generalized Young diagram, Osaka J. of Math. Vol.45 No.4 (2008), 1085-1120.

[5] K. Nakada and S. Okamura, Uniform generation of standard tableaux of a generalized Young diagram, submitted to Journal of Algebraic Combinatorics.

[6] K. Nakada and S. Okamura, An algorithm which generates linear extensions for a generalized Young diagram with uniform probability, DMTCS (Discrete Mathematics and Theoretical Computer Science), http://www.dmtcs.org/dmtcs-ojs/index.php/proceedings/article/view/dmAN0170/3216

[7] J. R. Stembridge, Minuscule elements of Weyl groups, J.Algebra 235 (2001), 722-743. 